\title{
Estimating severe irregularities of road ahead based on preceding vehicle responses
}

This paper was downloaded from TechRxiv (https://www.techrxiv.org).

\section{LICENSE}

CC BY-NC-SA 4.0

SUBMISSION DATE / POSTED DATE

20-01-2022 / 27-01-2022

\section{CITATION}

Li, Daofei; Xiao, Bin; Pan, Hao (2022): Estimating severe irregularities of road ahead based on preceding vehicle responses. TechRxiv. Preprint. https://doi.org/10.36227/techrxiv.18768230.v1

DOI

10.36227/techrxiv.18768230.v1 


\title{
Estimating severe irregularities of road ahead based on preceding vehicle responses
}

\author{
Daofei Li ${ }^{a} *$, Bin $\mathrm{Xiao}^{a}$ and $\mathrm{Hao} \mathrm{Pan}^{a}$ \\ ${ }^{a}$ Institute of Power Machinery and Vehicular Engineering, College of Energy Engineering, Zhejiang University, Hangzhou, China
}

\section{ARTICLE INFO}

Keywords:

Road irregularity estimation

Ride comfort

Driving safety

Vehicle dynamics

Kalman filter

Field data validation

\begin{abstract}
A B S T R ACT
Road irregularities, e.g. potholes or bulges, will cause discomfort, vehicle damages or even accidents, if not being carefully handled by drivers. For driving safety and comfort, especially in highly automated vehicles, there is a need for accurate and efficient way to estimate the road condition ahead in advance. Existing direct sensing based methods are difficult to give detailed irregularity information, while current response based approaches require either too many measurements or accurate system parameters to guarantee estimation performances. Therefore, using the information of preceding vehicle responses, a Kalman filter based algorithm to estimate severe road irregularities is proposed. The single degree of freedom vertical dynamics model is reorganized to reduce the measurement requirements of the Kalman filter. To cope with the limited information of the preceding vehicle, the model parameters are approximated according to suspension design theories. Simulation and field data validation shows that the estimation algorithm is effective and robust on different vehicles.
\end{abstract}

\section{Introduction}

Road roughness condition is an important factor that affects ride comfort and driving experience. Severe road irregularities, if drivers do not manage carefully enough, may damage vehicles or even cause accidents, which escalates a ride comfort problem into a safety-critical problem. For under-developed areas, it is common to encounter severe road damages, e.g. potholes or bulges. Therefore, there is a need for an efficient way to detect such challenging and dangerous road damages for both road users and maintainers.

On the other hand, advanced vehicle control technologies have been proposed for enhancing ride comfort and safety. For the purpose of improving comfort, active suspensions have already been used in mass production, especially for premium brands. Automated driving, including Advanced driving assistance system (ADAS) and highlyautomated driving, also aims to provide customers with better safety and ride comfort. However, current technologies of automated driving still focus mainly on how to handle driving tasks on smooth road, rather than that on rough road. Therefore, for proactive driving performances of automated vehicle, it is also necessary to know the rough road condition ahead in advance, and then to take precautions for improved safety and comfort.

There have been some researches on how to recognize or estimate road conditions ahead, which can be divided into two categories, i.e. direct-sensing based and responsebased. In the direct-sensing based approaches, the irregularity location, size or other features are estimated directly from the original information of road surface ahead. For example, images or point clouds are first collected with onboard cameras or lidars, and then they are processed using

\footnotetext{
*Corresponding author

@ dfli@zju.edu.cn (D. Li)

ORCID(s): 0000-0002-6909-0169 (D. Li)
}

computer-vision or machine learning methods for detecting and locating the road irregularities ahead. Rich information from lidar sensors is utilized to detect the road irregularity, such as optical deviation (Vupparaboina et al., 2015), scattering amplitude (Wang et al., 2016), and disparity histogram (Chen et al., 2017). With camera sensors, Li et al. (2018) and Fan et al. (2021) utilized disparity map to project 2dimensional stereo images to 3-dimensional world points, then fit the road surface, and classified the outlier points below the road surface as pothole regions. Ul Haq et al. (2019) considered the influence of vehicle moving on the images from stereo camera, which are preprocessed to reduce blur. They found high vehicle velocity has little effect on depth estimation, but increases the errors of pothole's area. Based on deep learning methods, the region of interest (ROI) can be first extracted from original images, then used to detect the potholes (Chen et al., 2020), and to estimate the pothole area (Chitale et al., 2020) and the depth (Kok et al., 2021). However, with such methods, the accuracy of detection or estimation depends heavily on the extracted ROI.

Direct-sensing based approaches can detect road irregularities before the vehicle arrives at. However, such approaches are difficult to tell the detailed information about the irregularities, such as depth and length. They are further limited by bad weather and poor illumination conditions, and can hardly detect abnormal road irregularities such as water-filled potholes. More importantly, when the sensor, either camera or lidar, is occluded by preceding vehicles, it is impossible to detect or locate the road irregularities.

Response-based approaches, in the indirect-sensing category, treat the road input estimation based on a vehicle vertical dynamics model, either explicit or implicit. The responses of vehicle passing over the irregular section, including body displacement, velocity and acceleration, are used as the available measurements. As an inverse dynamics problem, the task is to estimate the unknown road input 
from the system responses, given appropriate system parameters and measurements. Nguyen et al. (2019) classified the response-based approaches into three main kinds, i.e. model-based methods, data-driven methods, and frequency transfer functions. Data-driven methods require sufficient training data and are hard to handle different road types. Transfer function approaches mainly focus on the frequency domain characteristics of vertical dynamics, and can differentiate levels of stochastic road roughness. Compared to these two kinds, model-based methods are better choices to give more detailed information of road conditions ahead. Doumiati et al. (2011) assumed linear road input and used Kalman filter (KF) to estimate it from suspension deflection, vehicle body displacement and acceleration. An augmented $\mathrm{KF}$ was developed using the accelerator and gyroscope in smartphones as sensors (Zhao et al., 2019a). Zhong et al. (2019) and Zhao et al. (2019b) also used smartphone sensors to detect potholes by directly solving system differential equation, while the model parameters are fit using system responses during the underdamped period after passing over a pothole. Other model-based methods, including sliding mode observer (Imine and M'Sirdi, 2006; Rath et al., 2014), jump diffusion process estimator (Li et al., 2016), and Qparameterization (Doumiati et al., 2017), were proposed to estimate road profile and the geometric shape of irregularity.

However, these response-based approaches usually require accurate model parameters, including suspension stiffness and damping, sprung and/or unsprung masses, etc. In most researches, necessary measurements include body acceleration, while some need even more hard-to-obtain measurements, e.g. wheel centre height and suspension deflection. This implies that they may work well for experimental vehicles, but are difficult for real applications in normal vehicles. More importantly, these approaches using ego vehicle responses can not detect the irregularity in advance, and thus can not be applied for automated driving system to make better planning or control.

To address these needs, this paper proposes a novel algorithm for severe road irregularity estimation based on preceding vehicle responses. The main contributions are as follows. ( $i$ ) The single degree of freedom (DOF) suspension model is reorganized into a new form for road input estimation, which needs only a few measurements available from low-cost sensors. To handle the problem of limited preceding vehicle information, the model parameters are approximated according to vehicle suspension design theories. (ii) A Kalman-filter-based algorithm is proposed to estimate road irregularities on basis of preceding vehicle responses. Validation via simulation and field data tests shows its effectiveness and robustness on different preceding vehicles.

The rest of this paper is organized as follows. Section 2 introduces the vehicle vertical dynamics model and explains the rationality of suspension parameter approximation. Section 3 details the road input estimation algorithm design by using a new form of vehicle suspension model. Then the proposed algorithm is validated using both simulation and field data, in sections 4 and 5, respectively. Finally, section 6 concludes a summary of this research and gives potential applications and improvements in future work.

\section{Modeling}

\subsection{Vehicle vertical dynamics model}

Since only the motion of the preceding vehicle tail can be well-observed by ego vehicle sensors, this paper focuses on the rear suspension model. The road roughness input is assumed equal for both left and right tyres, and the coupling effect of front and rear axles is neglected. Theoretically, a rear suspension model, including the sprung mass (vehicle body) and unsprung mass (wheel-tyre), can describe the vertical dynamics of vehicle. The key parameters of such model should at least include suspension stiffness and damping coefficient, tyre stiffness and both masses, as shown in Fig. 1(a). However, in the focused problem of estimating road irregularities, the accurate parameters of the preceding vehicle, e.g., tyre vertical stiffness, are unavailable. Additionally, it is also difficult to observe the vertical movement of preceding vehicle tyres, while its body movement can be well-observed via camera or lidar sensors. Therefore, a single-DOF mass-spring-damper rear suspension model, as shown in Fig. 1(b), is adopted here.

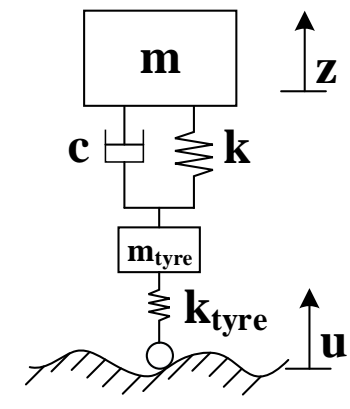

(a) 2-DOF model

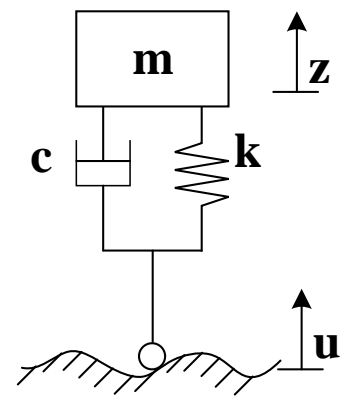

(b) 1-DOF model
Figure 1: Rear suspension model Eq. 1.

The vertical motion of vehicle body can be expressed as

$$
m \ddot{z}+c \dot{z}+k z=c \dot{u}+k u
$$

where $m$ is sprung mass; $c, k$ are the equivalent damping coefficient and spring stiffness of the suspension, respectively; $z, \dot{z}, \ddot{z}$ are the vertical displacement, velocity and acceleration of the sprung mass, respectively; $u, \dot{u}$ are road roughness input and its derivative to time, respectively.

A mass-normalized form can be derived by dividing both sides of Eq. 1 by $m$.

$$
\ddot{z}+c_{m} \dot{z}+k_{m} z=c_{m} \dot{u}+k_{m} u
$$

where $c_{m}=c / m, k_{m}=k / m$, are the mass-normalized damping coefficient and mass-normalized spring stiffness, respectively. 


\subsection{Model parameter approximation}

For real applications, the spring stiffness and damping coefficient should be reasonable to guarantee the precision of estimation algorithm. This is achieved by considering the basics of vehicle suspension design.

When designing the suspension of a vehicle, the first step is to choose the ride frequency $f$, which is defined as Eq. 3.

$$
f=\frac{1}{2 \pi} \sqrt{k / m}=\frac{1}{2 \pi} \sqrt{k_{m}}
$$

For passenger vehicles, the ride frequency $f$ is usually between 1.0-1.5 Hz (Reimpell et al. (2001), Yu (2018), Wong (2008)). Then the mass-normalized spring stiffness $k_{m}$ can be calculated when the ride frequency $f$ is designed, as shown in Eq. 4.

$$
k_{m}=(2 \pi f)^{2}
$$

By collecting the data of 38 passenger vehicle models, including sedans and SUVs, produced by different OEMs in recent three years, it is found that the ride frequency of rear suspension mainly falls between 1.3 and $1.5 \mathrm{~Hz}$, as shown in Fig. 2. The mean ride frequency is $1.39 \mathrm{~Hz}$, and its corresponding $k_{m}$ is $76.28 \mathrm{~N} /(\mathrm{kg} \cdot \mathrm{m})$, with standard deviation of $0.10 \mathrm{~Hz}$ and $11.28 \mathrm{~N} /(\mathrm{kg} \cdot \mathrm{m})$, respectively.

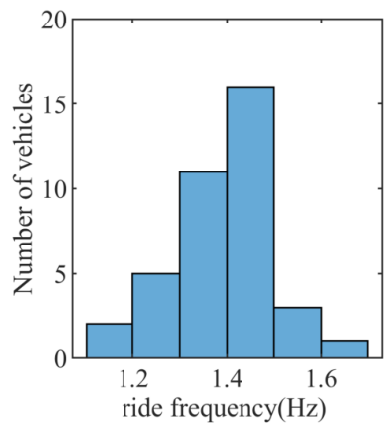

(a)

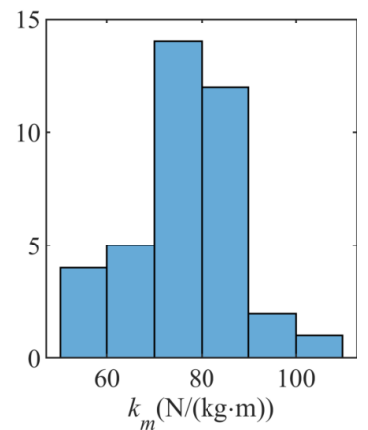

(b)
Figure 2: Statistical results of passenger vehicle ride frequency and calculated $k_{m}$ : (a) ride frequency of rear suspension; (b) $k_{m}$ of rear suspension

In the process of suspension damping design, the damping ratio $\zeta$ plays an important role in balancing response time and overshoot of vertical vibration under the excitation of road inputs. The damping ratio $\zeta$ is defined as Eq. 5, which is a function of $c_{m}$ and $k_{m}$. For passenger vehicles, the damping ratio ranges from 2.0 to 3.0 , and the most common value is 2.5 (Yu, 2018).

$$
\zeta=\frac{c}{2 \sqrt{m k}}=\frac{c / m}{2 \sqrt{k / m}}=\frac{c_{m}}{2 \sqrt{k_{m}}}
$$

Therefore, the mass-normalized damping coefficient $c_{m}$ can be obtained with known $k_{m}$ and $\zeta$, as shown in Eq. 6 .

\section{Severe road irregularities estimation based on KF}

\subsection{Kalman filter design}

The state equation of the single-DOF rear suspension model is rewritten as Eq. 7.

$$
\left[\begin{array}{c}
\ddot{z} \\
\dot{z}
\end{array}\right]=\left[\begin{array}{cc}
-c_{m} & -k_{m} \\
1 & 0
\end{array}\right]\left[\begin{array}{c}
\dot{z} \\
z
\end{array}\right]+\left[\begin{array}{cc}
c_{m} & k_{m} \\
0 & 0
\end{array}\right]\left[\begin{array}{c}
\dot{u} \\
u
\end{array}\right]
$$

It can be seen that the road input $u$ exists only in the equation of vertical acceleration of sprung mass $\ddot{z}$, which means $\ddot{z}$ needs to be observed to estimate the unknown road input in existing research (Doumiati et al., 2011; Zhao et al., 2019a; Zhong et al., 2019). However, the acceleration of the preceding vehicle is hard to be directly obtained by the sensors on the ego vehicle. Besides, the derivative of road input to time, i.e., $\dot{u}$, is also hard to handle in estimating the road input $u$.

Therefore, the original single-DOF rear suspension model Eq. 7 is reorganized into a new form in Eq. 8. This description is derived by utilizing the state space representation of linear differential equation which contains differentiated input. Compared with Eq. 7, the system state no longer contains the vertical acceleration $\ddot{z}$, and the differentiated road input $\dot{u}$ is also eliminated. That is to say, observing vertical displacement $z$ and velocity $\dot{z}$ is enough to estimate the unknown road input of the preceding vehicle. The displacement can be extracted from the front-view camera, and the velocity can be acquired by differentiating the displacement.

$$
\dot{\boldsymbol{X}}=\boldsymbol{A} \boldsymbol{X}+\boldsymbol{B} u
$$

where $\boldsymbol{X}=\left[\begin{array}{c}\dot{z}-c_{m} u \\ z\end{array}\right]$, is the system state, $\boldsymbol{A}=\left[\begin{array}{cc}-c_{m} & -k_{m} \\ 1 & 0\end{array}\right]$, $\boldsymbol{B}=\left[\begin{array}{c}k_{m}-c_{m}^{2} \\ c_{m}\end{array}\right]$.

The system measurement equation is shown as Eq. 9.

$$
\boldsymbol{Y}=\boldsymbol{C X}+\boldsymbol{D} u
$$

where $\boldsymbol{Y}=\left[\begin{array}{c}\dot{z} \\ z\end{array}\right], \boldsymbol{C}=\left[\begin{array}{ll}1 & 0 \\ 0 & 1\end{array}\right], \boldsymbol{D}=\left[\begin{array}{c}c_{m} \\ 0\end{array}\right]$.

With given $c_{m}$ and $k_{m}$, the state equation Eq. 8 is linear. To adapt to the form of Kalman filter, it is then discretized by introducing the state transition matrix $\boldsymbol{\Phi}=\boldsymbol{I}+\boldsymbol{A} t_{s}$ and the input matrix $\boldsymbol{\Gamma}=\boldsymbol{B} t_{s}$, where $\boldsymbol{I}$ is identity matrix, $t_{s}$ is the sampling time. Then the classical Kalman filter, including prediction and update process, can be described as Eq. 10 (Kalman, 1960).

$$
c_{m}=2 \zeta \sqrt{k_{m}}
$$




$$
\left\{\begin{aligned}
& \hat{\boldsymbol{X}}(k+1 \mid k)=\boldsymbol{\Phi} \hat{\boldsymbol{X}}(k \mid k)+\boldsymbol{\Gamma} u(k+1) \\
& \boldsymbol{P}(k+1 \mid k)=\boldsymbol{\Phi} \boldsymbol{P}(k \mid k) \boldsymbol{\Phi}^{T}+\boldsymbol{Q} \\
& \boldsymbol{Y}_{\boldsymbol{p r e}}(k+1 \mid k)=\boldsymbol{C} \hat{\boldsymbol{X}}(k+1 \mid k)+\boldsymbol{D} u(k+1) \\
& \boldsymbol{K}(k+1 \mid k)=\boldsymbol{P}(k+1 \mid k) \boldsymbol{C}^{T} \\
& \cdot\left(\boldsymbol{C P}(k+1 \mid k) \boldsymbol{C}^{T}+\boldsymbol{R}\right)^{-1} \\
& \hat{\boldsymbol{X}}(k+1 \mid k+1)=\hat{\boldsymbol{X}}(k+1 \mid k)+\boldsymbol{K}(k+1 \mid k) \\
& \cdot\left(\boldsymbol{Y}(k+1)-\boldsymbol{Y}_{\boldsymbol{p r e}}(k+1 \mid k)\right) \\
& \boldsymbol{P}(k+1 \mid k+1)=(\boldsymbol{I}-\boldsymbol{K}(k+1 \mid k) \boldsymbol{C}) \boldsymbol{P}(k+1 \mid k)
\end{aligned}\right.
$$

where $\hat{\boldsymbol{X}}(k+1 \mid k)$ and $\boldsymbol{P}(k+1 \mid k)$ are predicted state estimate and predicted estimate covariance, respectively; $\boldsymbol{Y}_{\text {pre }}(k+$ $1 \mid k)$ is the predicted measurement; $\boldsymbol{K}(k+1 \mid k)$ is the Kalman gain; $\hat{\boldsymbol{X}}(k+1 \mid k+1)$ and $\boldsymbol{P}(k+1 \mid k+1)$ are the updated state estimate and predicted estimate covariance, respectively; $\boldsymbol{Q}$ and $\boldsymbol{R}$ are the covariance of process noise and measurement noise, respectively.

The unknown road input is estimated as Eq. 11, which can be derived from Eq. 9 .

$$
\hat{u}(k \mid k)=\boldsymbol{D}_{\boldsymbol{R}}(\boldsymbol{Y}(k)-\boldsymbol{C} \hat{\boldsymbol{X}}(k \mid k))
$$

where $\boldsymbol{D}_{\boldsymbol{R}}=\left[\begin{array}{ll}0 & 1 / c_{m}\end{array}\right]$.

With a relatively short sampling time, the road inputs of two adjacent time steps may not differ greatly. Therefore, the estimated road input at time $k$, i.e. $\hat{u}(k \mid k)$, is assumed to be the input of time $k+1$, i.e. $u(k+1)$, during the state and measurement prediction steps of the classical Kalman filter. The state prediction in Eq. 10 can be rewritten as Eq. 12 by substituting Eq. 11 into the first equation in Eq. 10.

$$
\begin{aligned}
\hat{\boldsymbol{X}}(k+1 \mid k) & =\boldsymbol{\Phi} \hat{\boldsymbol{X}}(k \mid k)+\boldsymbol{\Gamma} \boldsymbol{D}_{\boldsymbol{R}}(\boldsymbol{Y}(k)-\boldsymbol{C} \hat{\boldsymbol{X}}(k \mid k)) \\
& =\left(\boldsymbol{\Phi}-\boldsymbol{\Gamma} \boldsymbol{D}_{\boldsymbol{R}} \boldsymbol{C}\right) \hat{\boldsymbol{X}}(k \mid k)+\boldsymbol{\Gamma} \boldsymbol{D}_{\boldsymbol{R}} \boldsymbol{Y}(k) \\
& =\boldsymbol{M} \hat{\boldsymbol{X}}(k \mid k)+\boldsymbol{N} \boldsymbol{Y}(k) \\
\hat{\boldsymbol{X}}(k \mid k) & =\boldsymbol{M}^{-1}(\hat{\boldsymbol{X}}(k+1 \mid k)-\boldsymbol{N} \boldsymbol{Y}(k))
\end{aligned}
$$

Substituting Eq. 11 and Eq. 13 into the third equation of Eq. 10, the measurement prediction equation can be rewritten as Eq. 14.

$$
\begin{aligned}
\boldsymbol{Y}_{\text {pre }}(k+1 \mid k)= & \boldsymbol{C} \hat{\boldsymbol{X}}(k+1 \mid k)+\boldsymbol{D} \boldsymbol{D}_{\boldsymbol{R}}(\boldsymbol{Y}(k)-\boldsymbol{C} \hat{\boldsymbol{X}}(k \mid k)) \\
= & \left(\boldsymbol{C}-\boldsymbol{D} \boldsymbol{D}_{\boldsymbol{R}} \boldsymbol{C} \boldsymbol{M}^{-\mathbf{1}}\right) \hat{\boldsymbol{X}}(k+1 \mid k) \\
& +\boldsymbol{D} \boldsymbol{D}_{\boldsymbol{R}}\left(\boldsymbol{I}+\boldsymbol{C} \boldsymbol{M}^{-\mathbf{1}} \boldsymbol{N}\right) \boldsymbol{Y}(k) \\
= & \boldsymbol{H} \hat{\boldsymbol{X}}(k+1 \mid k)+\boldsymbol{G} \boldsymbol{Y}(k)
\end{aligned}
$$

Then a new form of Kalman filter is given in Eq. 15.

$$
\left\{\begin{aligned}
\hat{\boldsymbol{X}}(k+1 \mid k)=\boldsymbol{M} \hat{\boldsymbol{X}}(k \mid k)+\boldsymbol{N} \boldsymbol{Y}(k) \\
\boldsymbol{P}(k+1 \mid k)=\boldsymbol{M} \boldsymbol{P}(k \mid k) \boldsymbol{M}^{T}+\boldsymbol{Q} \\
\boldsymbol{Y}_{\boldsymbol{p r e}}(k+1 \mid k)=\boldsymbol{H} \hat{\boldsymbol{X}}(k+1 \mid k)+\boldsymbol{G} \boldsymbol{Y}(k) \\
\boldsymbol{K}(k+1 \mid k)=\boldsymbol{P}(k+1 \mid k) \boldsymbol{H}^{T} \\
\cdot\left(\boldsymbol{H} \boldsymbol{P}(k+1 \mid k) \boldsymbol{H}^{T}+\boldsymbol{R}\right)^{-1} \\
\hat{\boldsymbol{X}}(k+1 \mid k+1)=\hat{\boldsymbol{X}}(k+1 \mid k)+\boldsymbol{K}(k+1 \mid k) \\
\cdot\left(\boldsymbol{Y}(k+1)-\boldsymbol{Y}_{\boldsymbol{p r e}}(k+1 \mid k)\right) \\
\boldsymbol{P}(k+1 \mid k+1)=(\boldsymbol{I}-\boldsymbol{K}(k+1 \mid k) \boldsymbol{H}) \boldsymbol{P}(k+1 \mid k)
\end{aligned}\right.
$$

The overall structure of the proposed KF algorithm is shown as Fig. 3. Comparing Eq. 15 with Eq. 10, the only difference is that the model input $u(k+1)$ is replaced by the measurement of last time step $\boldsymbol{Y}(k)$ in Eq. 15, and the coefficient matrices change correspondingly. Therefore, road input estimation based on preceding vehicle responses can be achieved with relatively easy-to-obtain measurements.

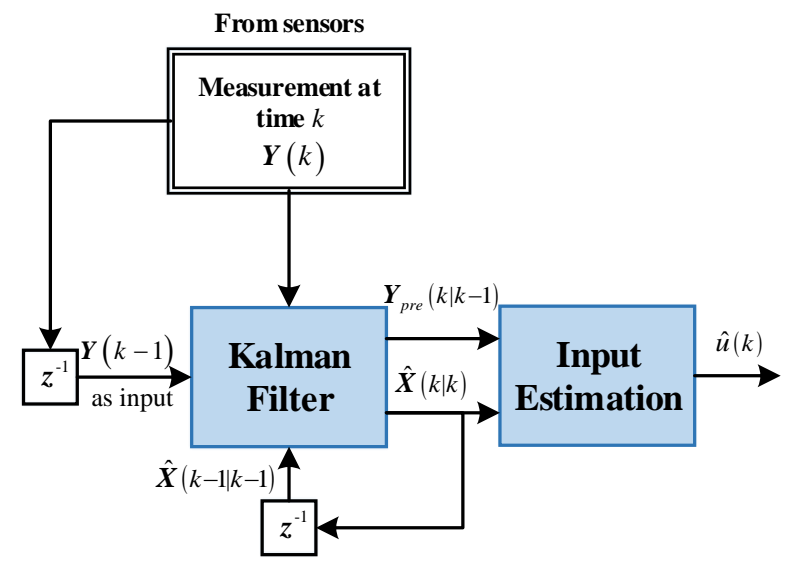

Figure 3: Structure of proposed KF

\subsection{Influence of suspension bump stop}

Since severe road irregularities are considered here, the suspension deflection may reach its compression or rebound stroke limit, i.e. bump stop. The bump stop may be hit and the suspension stiffness surges, which will significantly affect the estimation accuracy. Therefore, the model built in subsection 2.1 should be improved to cover such case.

Based on the single-DOF model, the suspension deflection is derived as $u-z$, since the tyre is regarded as a rigid body, compared to vehicle suspension. The bump stop is regarded as a spring with a larger stiffness here. Then a piecewise suspension model is adopted, with different stiffnesses before and after threshold of suspension deflection, as shown in Eq. 16. Once the suspension deflection exceeds the threshold $d_{0}$, it can be considered that the bump stop is hit and the stiffness of the suspension increases. $k_{s p r}$ refers to the massnormalized stiffness of the suspension spring, and $k_{b s}$ refers to the mass-normalized stiffness of the bump stop. 
Estimating severe irregularities of road ahead based on preceding vehicle responses

$$
k_{m}= \begin{cases}k_{s p r}, & |u-z| \leqslant d_{0} \\ k_{s p r}+k_{b s}, & |u-z|>d_{0}\end{cases}
$$

\section{Validation via simulation}

\subsection{Benchmark algorithm}

Discrete Kalman filter with unknown input (DKF-UI) is chosen as the benchmark algorithm. It was first proposed for structural damage identification (Yang et al., 2007), and has been used to estimate road roughness (Kang et al., 2019).

To meet the requirements of DKF-UI, the system state and measurement equations are revised to Eq. 17 and Eq. 18, respectively, which are slightly different from Eq. 8 and Eq. 9. In Eq. 17, as a revised form of Eq. 7, $F=c_{m} \dot{u}+k_{m} u$ can be viewed as the vertical force between tyre and road.

$$
\begin{aligned}
{\left[\begin{array}{c}
\ddot{z} \\
\dot{z}
\end{array}\right] } & =\left[\begin{array}{cc}
-c_{m} & -k_{m} \\
1 & 0
\end{array}\right]\left[\begin{array}{l}
\dot{z} \\
z
\end{array}\right]+\left[\begin{array}{l}
1 \\
0
\end{array}\right] F \\
\dot{\boldsymbol{X}} & =\boldsymbol{A} \boldsymbol{X}+\boldsymbol{B}^{*} F \\
{\left[\begin{array}{c}
\ddot{z} \\
\dot{z} \\
z
\end{array}\right] } & =\left[\begin{array}{cc}
-c_{m} & -k_{m} \\
1 & 0 \\
0 & 1
\end{array}\right]\left[\begin{array}{l}
\dot{z} \\
z
\end{array}\right]+\left[\begin{array}{l}
1 \\
0 \\
0
\end{array}\right] F \\
\boldsymbol{Y}^{*} & =\boldsymbol{C}^{*} \boldsymbol{X}+\boldsymbol{D}^{*} F
\end{aligned}
$$

With Eq. 17 and Eq. 18, the DKF-UI algorithm, described as Eq. 19, is used to estimate the tyre-road force $F$. Then, the unknown road input $u$ is obtained by solving $\hat{F}=c_{m} \dot{u}+k_{m} u$.

$$
\left\{\begin{array}{c}
\hat{\boldsymbol{X}}(k+1 \mid k)=\boldsymbol{\Phi} \hat{\boldsymbol{X}}(k \mid k)+\boldsymbol{\Gamma}^{*} \hat{\boldsymbol{F}}(k) \\
\boldsymbol{P}^{*}(k+1 \mid k)=\boldsymbol{\Phi} \boldsymbol{P}^{*}(k \mid k) \boldsymbol{\Phi}^{T}+\boldsymbol{Q} \\
\boldsymbol{K}^{*}(k+1 \mid k)=\boldsymbol{P}^{*}(k+1 \mid k) \boldsymbol{C}^{* T} \\
\cdot\left[\boldsymbol{C}^{*} \boldsymbol{P}^{*}(k+1 \mid k) \boldsymbol{C}^{* T}+\boldsymbol{R}^{*}\right]^{-1} \\
\boldsymbol{S}(k+1)=\left[\boldsymbol{D}^{* \boldsymbol{T}} \boldsymbol{R}^{*-1}\left(\boldsymbol{I}-\boldsymbol{C}^{*} \boldsymbol{K}(k+1 \mid k)\right) \boldsymbol{D}^{*}\right]^{-1} \\
\hat{\boldsymbol{F}}(k+1)=\boldsymbol{S}(k+1) \boldsymbol{D}^{* T} \boldsymbol{R}^{*-1}\left[\boldsymbol{I}-\boldsymbol{C}^{*} \boldsymbol{K}(k+1)\right] \\
\cdot\left[\boldsymbol{Y}^{*}(k+1)-\boldsymbol{C}^{*} \hat{\boldsymbol{X}}(k+1 \mid k)\right] \\
\hat{\boldsymbol{X}}(k+1 \mid k+1)=\hat{\boldsymbol{X}}(k+1 \mid k)+\boldsymbol{K}^{*}(k+1 \mid k) \\
\cdot\left[\boldsymbol{Y}^{*}(k+1)-\boldsymbol{C}^{*} \hat{\boldsymbol{X}}(k+1 \mid k)\right. \\
\left.-\boldsymbol{D}^{*} \hat{\boldsymbol{F}}(k+1)\right] \\
\boldsymbol{P}^{*}(k+1 \mid k+1)=\left[\boldsymbol{I}+\boldsymbol{K}^{*}(k+1 \mid k) \boldsymbol{D}^{*} \boldsymbol{S}(k+1)\right. \\
\left.\cdot \boldsymbol{D}^{* \boldsymbol{T}} \boldsymbol{R}^{*-1} \boldsymbol{C}^{*}\right] \\
\cdot\left[\boldsymbol{I}-\boldsymbol{K}^{*}(k+1 \mid k) \boldsymbol{C}^{*}\right] \boldsymbol{P}^{*}(k+1 \mid k)
\end{array}\right.
$$

where $\boldsymbol{\Gamma}^{*}=\boldsymbol{B}^{*} t_{s}$.

It should be noted that two constraints must be satisfied to implement DKF-UI (Yang et al., 2007; Kang et al., 2019):

(i) $B^{*} \neq O, D^{*} \neq O$
Table 1

Rear suspension paramters of simulation vehicle

\begin{tabular}{ll}
\hline Parameter (unit) & Physical value \\
\hline$m(\mathrm{~kg})$ & 664.25 \\
$k(\mathrm{~N} / \mathrm{m})$ & 50000 \\
$c(\mathrm{~N} \cdot \mathrm{s} / \mathrm{m})$ & 2882.85 \\
$k_{s p r}(\mathrm{~N} /(\mathrm{kg} \cdot \mathrm{m}))$ & 75.27 \\
$\left.k_{s p r}+k_{b s}(\mathrm{~N} / \mathrm{kg} \cdot \mathrm{m})\right)$ & 200 \\
$d_{0}(\mathrm{~m})$ & 0.08 \\
$c_{m}(\mathrm{~N} \cdot \mathrm{s} /(\mathrm{kg} \cdot \mathrm{m}))$ & 4.34 \\
$\zeta$ & 0.25 \\
\hline
\end{tabular}

(ii) The number of measurements should be larger than the number of unknown inputs.

This means the vertical acceleration $\ddot{z}$ must be observed to satisfy constraint (i), since only the equation of $\ddot{z}$ contains the unknown input.

\subsection{Simulation setups}

Simulation experiments based on IPG Carmaker are carried out for preliminary validation of the proposed algorithm. A B-class passenger vehicle is chosen here, with rear suspension parameters shown in Table. 1.

Fig. 4 shows the cross-section view of irregularities as the road input in the simulation, with mamximum height or depth $h_{o b s}$ set to $\pm 0.15, \pm 0.10$ and $\pm 0.05 \mathrm{~m}$ for six cases. Both positive and negative obstacles are considered. A positive obstacle, or a road bulge, is the section with raised height above road plane, while a negative obstacle means a pothole with lowered height below road plane. The road excitations for both rear tyres are the same, as required by the model assumptions. In simulation, the vehicle drives at different constant velocities from 10 to $30 \mathrm{~km} / \mathrm{h}$, with an interval of $5 \mathrm{~km} / \mathrm{h}$.

positive obstacle (bulge)

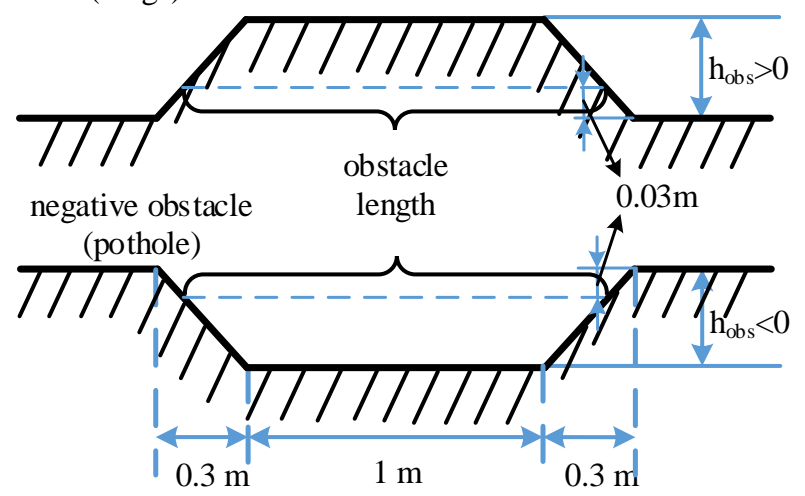

Figure 4: Cross-section view of irregularities in simulation

For system measurements, the vertical displacement of the rear axle is calculated from the vertical movement of vehicle centre of mass and body pitch angle, which are 


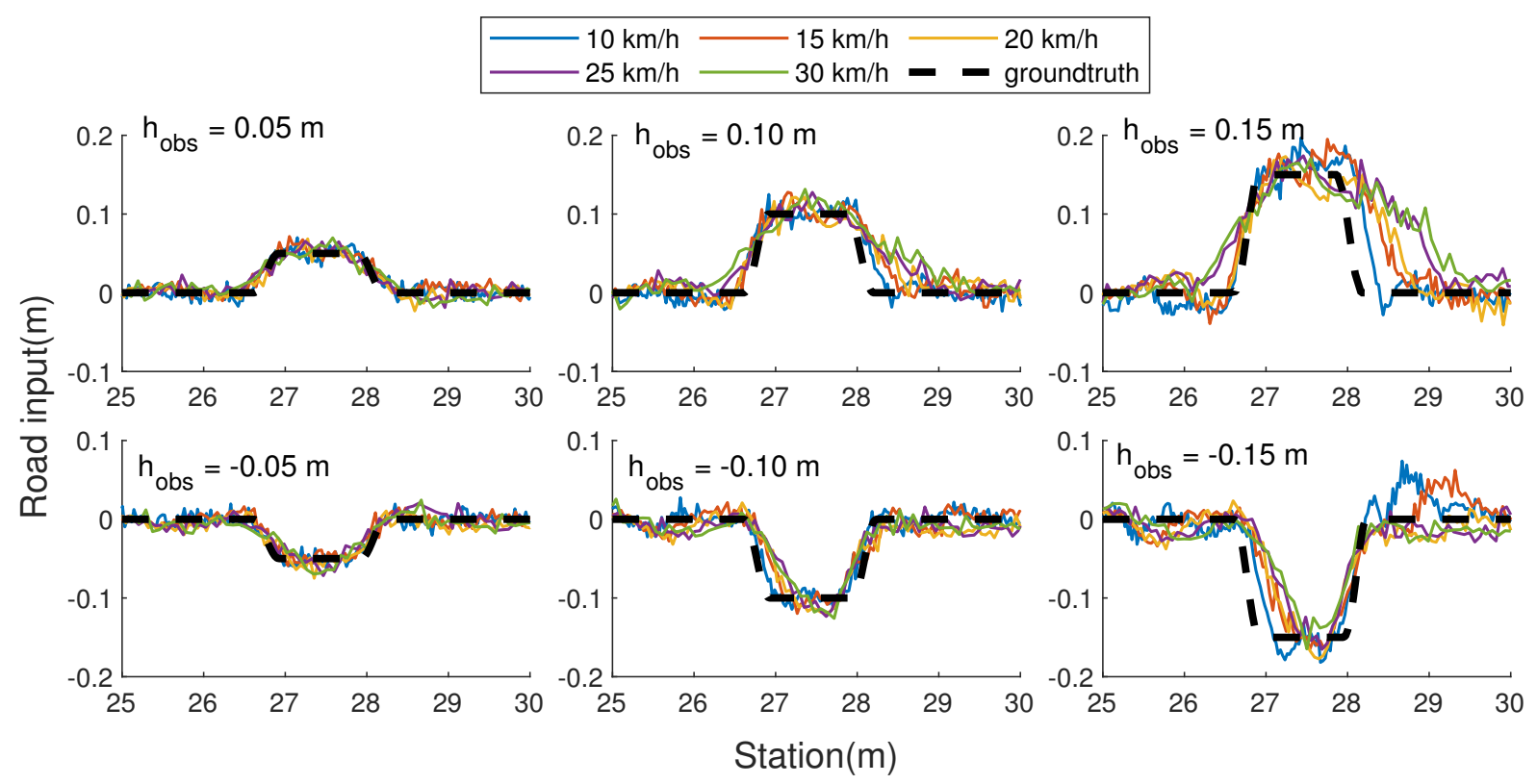

Figure 5: Estimation results of proposed algorithm

Table 2

Sensor noise variances in simulation

\begin{tabular}{lll}
\hline Signal & Units & Variance \\
\hline$z$ & $\mathrm{~m}$ & $1 \times 10^{-4}$ \\
$\dot{z}$ & $\mathrm{~m} / \mathrm{s}$ & $6.25 \times 10^{-4}$ \\
$\ddot{z}$ & $\mathrm{~m} / \mathrm{s}^{2}$ & 1 \\
\hline
\end{tabular}

acquired from IPG CarMaker. The vertical velocity is obtained by differentiating the displacement, and the vertical acceleration needed for DKF-UI is obtained by differentiating the velocity. Besides, to simulate the sensor noises, normally distributed noises with zero-means are added to the measurements, as shown in Table. 2.

\subsection{Simulation results}

The estimation results of proposed algorithm and DKFUI are shown in Fig. 5 and Fig. 6, respectively. The thick dashed lines are the groundtruth, and the thin solid lines are the estimation results at different velocities.

For qualitative analysis, it can be found that both algorithms can estimate the groundtruth shape of road irregularities, especially when $h_{o b s}= \pm 0.05, \pm 0.10 \mathrm{~m}$. The estimation for the height of road irregularities $h_{o b s}$ is accurate under different velocities, even when the $h_{o b s}$ reaches $0.15 \mathrm{~m}$. However, the obstacle length estimation becomes worse with the increase of velocity.

To quantitively analyze the estimation results, the maximum height error and length error are introduced, where maximum height error is the maximum error between the estimation and groundtruth of the peak irregularity. To discriminate the irregularity from stochastic roughness, a height threshold of $0.03 \mathrm{~m}$ is chosen. It means the road irregularity is distinguished only when the estimated road input magnitude is large enough, i.e., $|\hat{u}| \geq 0.03 \mathrm{~m}$. As shown in Fig. 4, to define the length of irregular section, the first and the last stations with $|\hat{u}| \geq 0.03 \mathrm{~m}$ are chosen as the beginning and end of the road irregularity, respectively.

As shown in Fig. 7, the maximum height error of both algorithms is mainly between 0.01 to $0.03 \mathrm{~m}$, and varies slightly with velocity. The max height error of DKF-UI is slightly better than the proposed algorithm. However, the length error varies greatly with velocity when $h_{o b s}$ is large, which is the most obvious when $h_{o b s}=0.10$ and 0.15 $\mathrm{m}$. Compared with the groundtruth, the estimation shows a delay effect, which aggravates with increasing velocity when the vehicle leaves the bulge. The same phenomenon can also be found when the vehicle enters the pothole. This is mainly because the tyre leaves the ground when the vehicle travels from high plane to low, while the vertical dynamics model adopted assumes that the tyre directly contacts with the ground. Therefore, the estimation results actually reflect the tyre displacement, and thus the delay effct occurs when the tyre leaves the ground, which is more obvious at high velocity. This explains why the length estimation is longer than groundtruth in positive obstacle, and is shorter in negative obstacle in Fig. 8.

To summarize, simulation results show that both algorithms are effective in estimating the depth under different velocities. But with fewer and easier-to-obtain measurements than that of DKF-UI, the proposed algorithm can perform almost the same as DKF-UI in both depth and length estimations of road irregularities.

\section{Field data validation}

\subsection{Data collection and preprocessing}

To further validate the robustness and effectiveness of the algorithm on different vehicles, field data are collected 


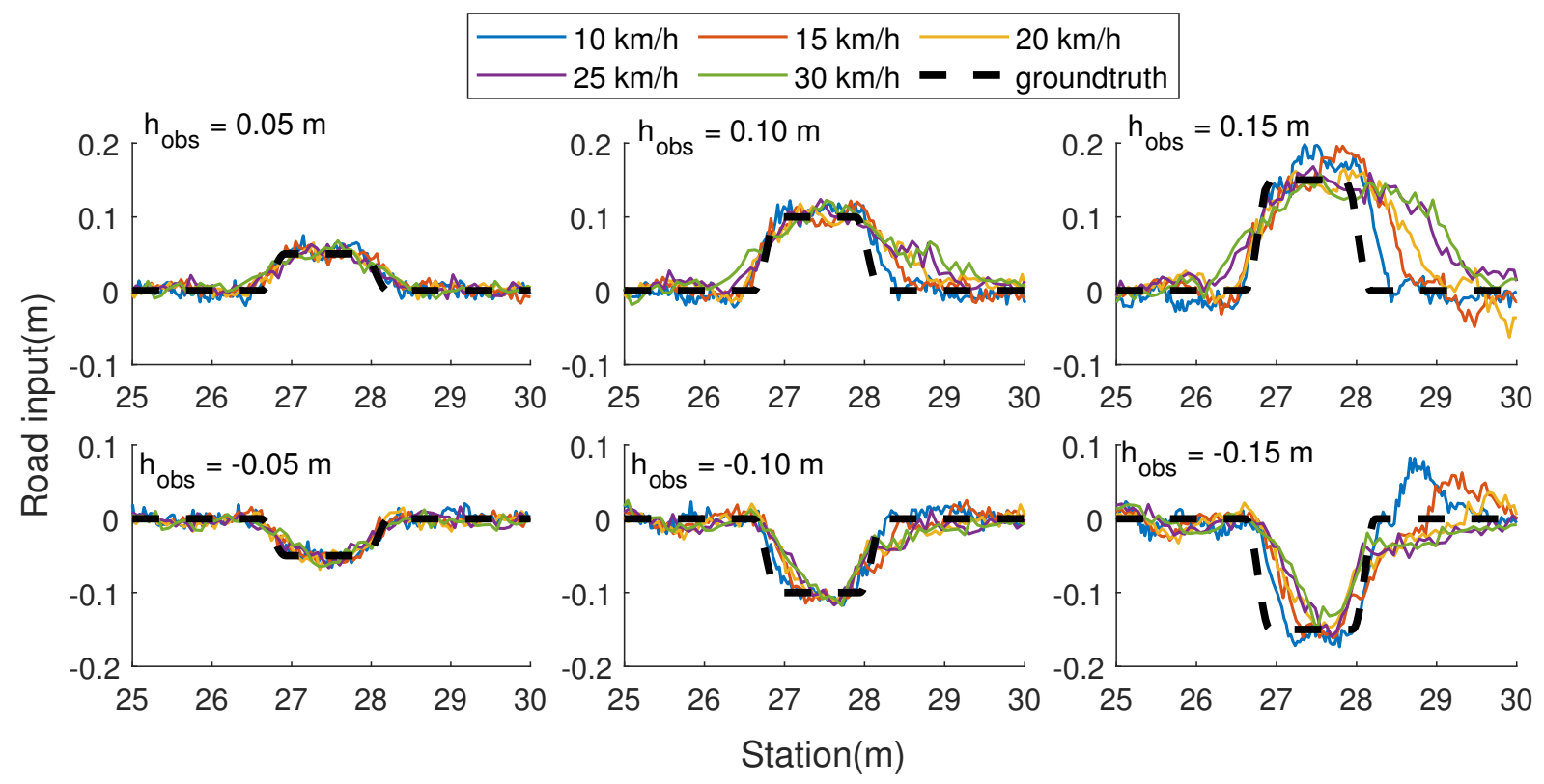

Figure 6: Estimation results of DKF-UI

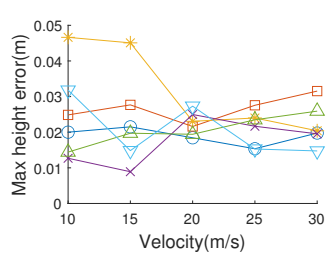

(a)

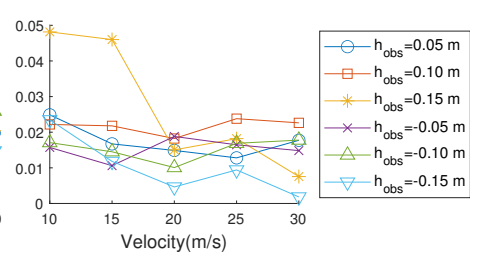

(b)
Figure 7: Max height errors: (a) Proposed algorithm; (b) DKFUI

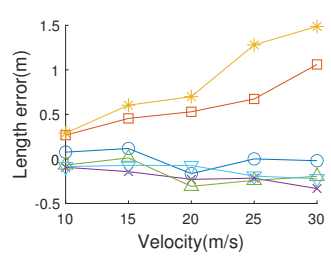

(a)

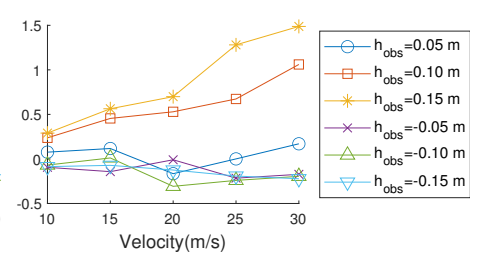

(b)
Figure 8: Length errors: (a) Proposed algorithm; (b) DKF-UI

in a road section with a pothole. The dynamic responses of different vehicles passing by are recorded using a camera at the roadside, with $1920 \times 1080$ resolution at 60 frames per second. In total, 76 vehicles are selected for validation.

As shown in Fig. 9, for the groundtruth, the real depth of the pothole is measured by a solid-state lidar Livox Horizon, which features non-repetitive horizontal scanning patterns, with integration time of 3 seconds. The road surface point cloud of the considered lane section is shown in Fig. 10. It can be noticed that the pothole depth varies along with the lateral direction. This means the road input will be different when a vehicle passes at different lateral positions. To simplify, the depth is averaged in lateral direction so that it only varies in the longitudinal direction. The averaged road input is shown in Fig. 11. The maximum depth of averaged road input is about $0.089 \mathrm{~m}$.

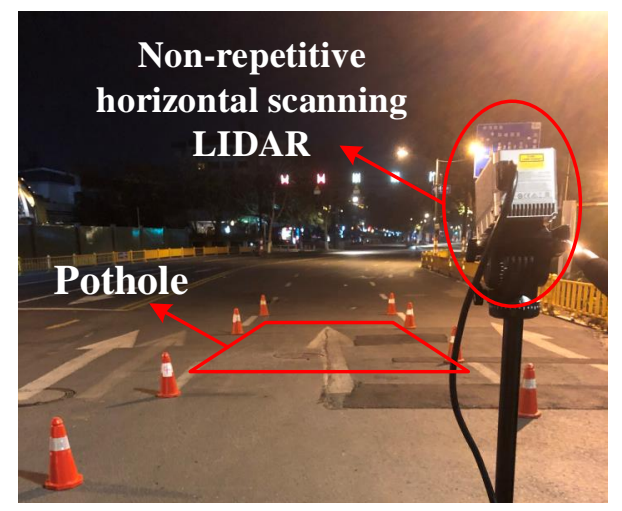

Figure 9: Groundtruth collection

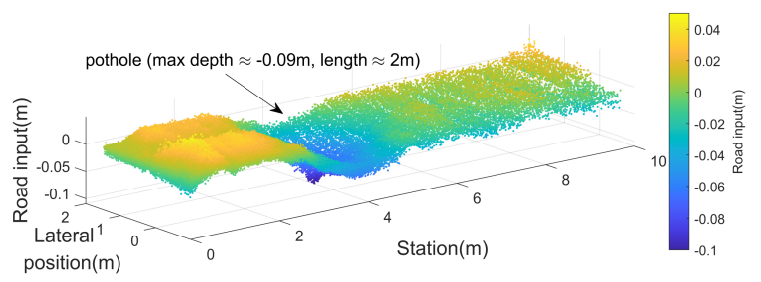

Figure 10: Point cloud of the target lane with pothole

For data preprocessing, it is assumed that the distance between the camera and vehicles passing the pothole remains the same. Then the real-world vertical displacement is proportional to the difference of pixel coordinates in the image captured by the camera with no pitch angle. Therefore, the vertical displacement of the sprung mass can be obtained 


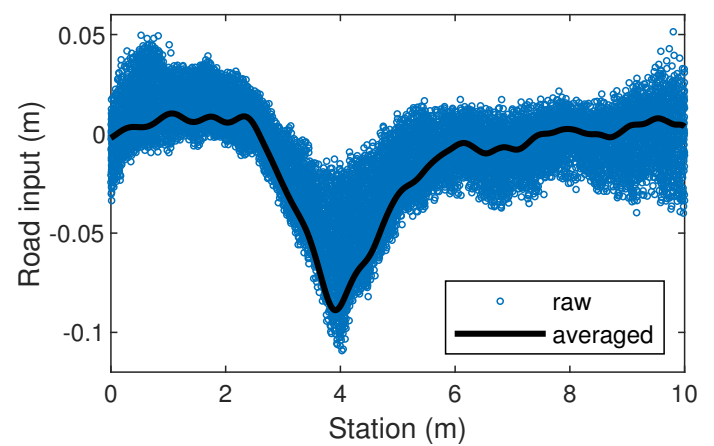

Figure 11: Cross-section view of road input averaged along lateral direction

by tracking a feature point on the vehicle body. Here, the taillights are chosen as the feature points, and are tracked by CSRT tracker (Gong et al., 2019) in OpenCV, as shown in Fig. 12. The coordinates of the tracking box centre are used to calculate the real-world vertical displacement of vehicle body. The vertical velocity is obtained by differentiating the displacement, and the vertical acceleration is obtained by differentiating the velocity. The vertical displacement and velocity are filtered by a Savitzky-Golay filter before being differentiated.

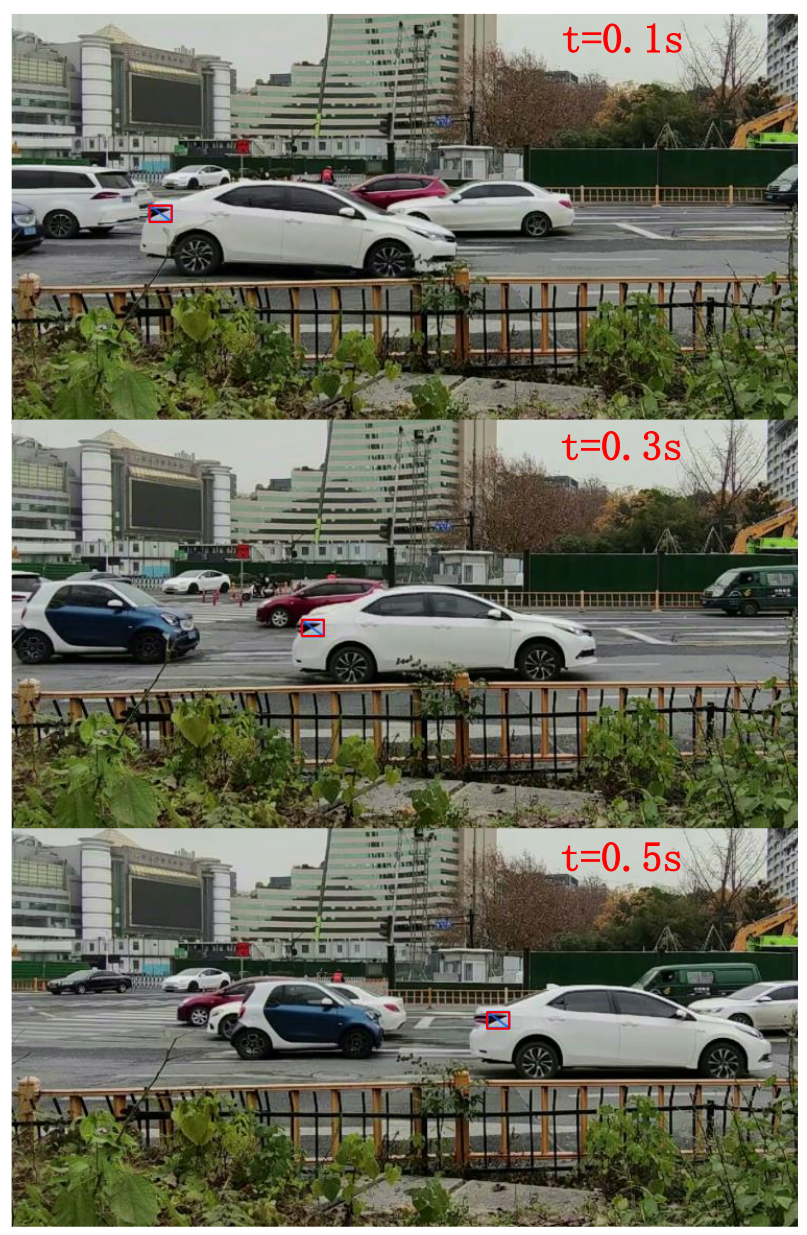

Figure 12: Taillights tracking

\subsection{Results}

Without loss of generality, the mass-normalized suspension parameters are chosen as the mean value of the statistical results in subsection 2.2, i.e., $k_{s p r}=76.28 \mathrm{~N} /(\mathrm{kg} \cdot \mathrm{m})$, $k_{s p r}+k_{b s}=150 \mathrm{~N} /(\mathrm{kg} \cdot \mathrm{m}), c_{m}=4.37(\mathrm{~N} \cdot \mathrm{s}) /(\mathrm{kg} \cdot \mathrm{m})$.

Fig. 13 shows the estimation results of all 76 vehicles of both algorithms. The dashed lines are the estimation results of every single vehicle, and the thick solid line is the averaged road input, i.e. the groundtruth. It can be seen that for the first estimation, there is an obvious jump from zero in height between the first two time steps in Fig. 13(a). This is because the initial states in KF estimation are set to zero vector, while the real vehicle states, especially the vertical velocity, are not zero. For the pothole at the station of $4 \mathrm{~m}$, it can be found that most estimations can approximately reflect the pothole depth and cross-section shape.

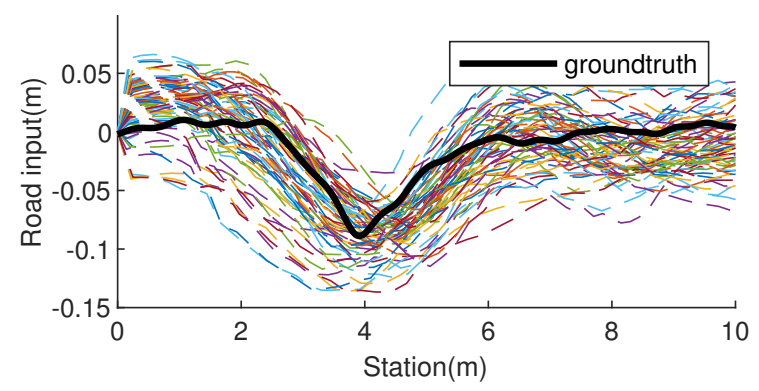

(a)

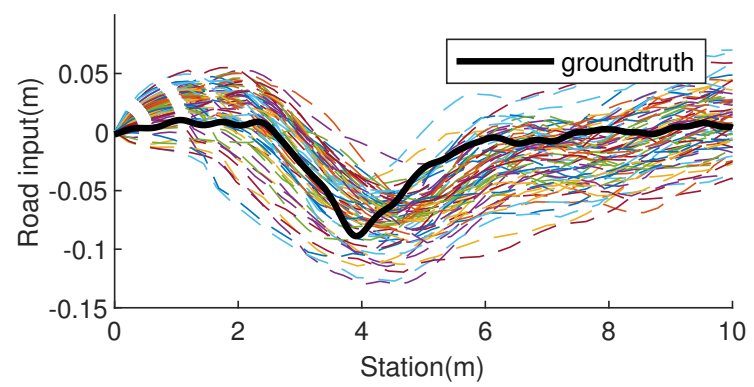

(b)

Figure 13: All estimation results of field data: (a) proposed algorithm; (b) DKF-UI

For overall evaluation of both algorithms, the averaged results of all estimations are shown in Fig. 14, and the statistical results are shown in Fig. 15. It can be found that both estimation algorithms performs significantly better than merely observing the vertical displacement of sprung mass $z$, in estimating the length and depth of road irregularities. The averaged result of DKF-UI shows underestimated pothole depth and also location offset in the longitudinal direction. This is mainly because DKF-UI needs an extra measurement $\ddot{z}$, which is obtained by double differentiating. The noise of the original measurement $z$ in real world data is greatly amplified by differentiating, and thus affects the estimation results. This is also why such phenomenon does not occur in simulation results.

Comparing with DKF-UI, the proposed algorithm can better estimate the depth, length, and location of the pothole, as shown in Table. 3. The statistics in Fig. 15 further show 
Table 3

Averaged length and depth estimation of two algorithms

\begin{tabular}{lll}
\hline & $\begin{array}{l}\text { Max depth }(\mathrm{m}) \\
\text { at station }(\mathrm{m})\end{array}$ & Length $(\mathrm{m})$ \\
\hline Groundtruth & 0.089 at 3.92 & 2.00 \\
Proposed algorithm & 0.084 at 4.23 & 2.80 \\
DKF-UI & 0.073 at 4.60 & 2.92 \\
\hline
\end{tabular}

that the proposed algorithm can accurately estimate the pothole depth, while DKF-UI tend to underestimate the pothole depth.
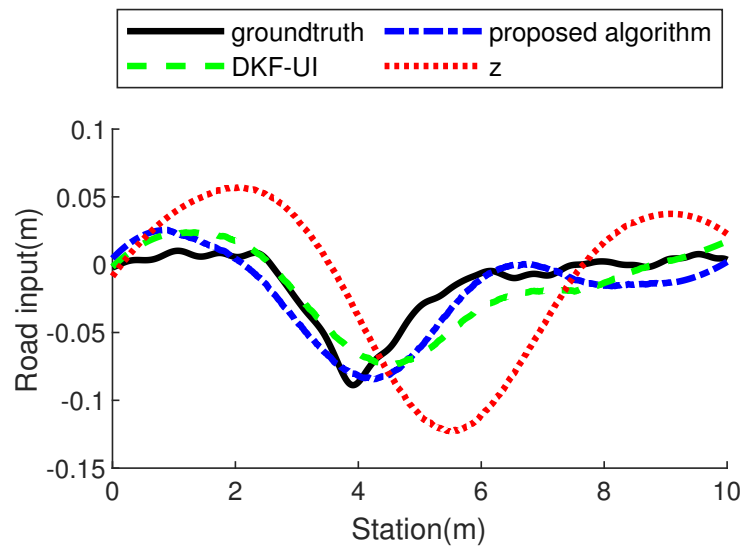

Figure 14: Averaged results of all estimations

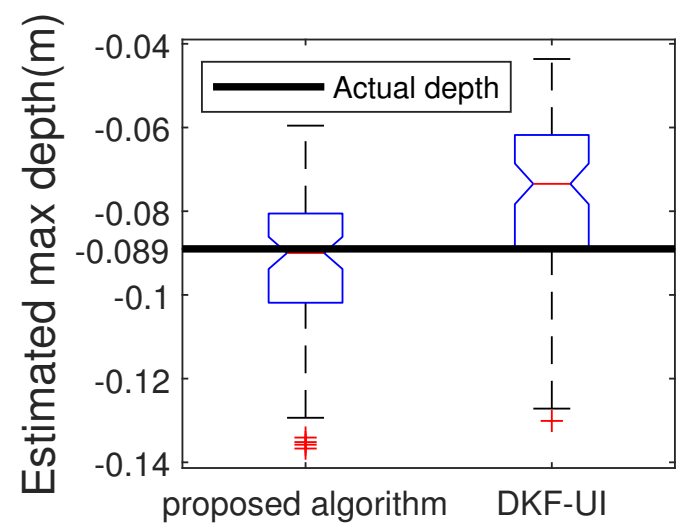

Figure 15: Statistical results of estimated max depth

\section{Conclusions}

This paper proposes a Kalman filter based road irregularity estimation algorithm on basis of preceding vehicle responses. A new form of single-DOF suspension model is adopted, which reduces the number of necessary measurements in estimation. To solve the problem of unknown parameters of the preceding vehicle, statistics of various vehicles from different OEMs are made to give an approximation. Simulation results reflect the effectiveness of the algorithm in both depth and length estimation under different vehicle velocities, with an accurate suspension model. Besides, field data validation shows the robustness of the algorithm on different vehicles. The comparison with the benchmark algorithm underlines the advantage of fewer measurements, which is less affected by the sensor noises. The proposed algorithm may work as promising solution to road irregularity identification and estimation with low-cost sensors. Potential applications include (pro)active suspension control, enhanced driving decision for better comfort and safety, etc. It may also be deployed in smart road system, e.g. via roadside edge computing, which can detect road damages or big debris and ignite warnings for road users and maintainers.

This work is a part of an on-going research on proactive planning algorithm for automated driving. There are still some improvements that can be done in future work.

(i) Sensing of the preceding vehicle is done by sidecamera in field data validation, which should be further validated by a front-view camera or a lidar on the ego vehicle. Motion compensation and estimation of distance to the preceding vehicle should be solved to acquire the vertical displacement.

(ii) Further statistics on suspension parameters of different vehicle types can be collected to give a more accurate approximation according to the type of the preceding vehicle.

\section{Acknowledgements}

The authors sincerely appreciated Siyuan Lin for his advices on model reorganization, and Linhui Chen for his help in preparing the video abstract.

Funding: This work was supported by Department of Science and Technology of Zhejiang [grant number 2022C$01241]$.

\section{References}

Chen, H., Yao, M., Gu, Q., 2020. Pothole detection using location-aware convolutional neural networks. International Journal of Machine Learning and Cybernetics 11, 899-911. doi:10.1007/s13042-020-01078-7.

Chen, L., Yang, J., Kong, H., 2017. Lidar-histogram for fast road and obstacle detection, in: 2017 IEEE International Conference on Robotics and Automation (ICRA), Singapore. pp. 1343-1348. doi:10.1109/ICRA. 2017.7989159.

Chitale, P.A., Kekre, K.Y., Shenai, H.R., Karani, R., Gala, J.P., 2020. Pothole detection and dimension estimation system using deep learning (YOLO) and image processing, in: 2020 35th International Conference on Image and Vision Computing New Zealand (IVCNZ), IEEE, Wellington, New Zealand. pp. 1-6. doi:10.1109/IVCNZ51579.2020.9290547.

Doumiati, M., Martinez, J., Sename, O., Dugard, L., Lechner, D., 2017. Road profile estimation using an adaptive Youla-Kučera parametric observer: Comparison to real profilers. Control Engineering Practice 61, 270-278. doi:10.1016/j. conengprac. 2015.12.020.

Doumiati, M., Victorino, A., Charara, A., Lechner, D., 2011. Estimation of road profile for vehicle dynamics motion: Experimental validation, in: Proceedings of the 2011 American Control Conference, pp. 5237-5242. doi:10.1109/ACC. 2011.5991595.

Fan, R., Ozgunalp, U., Wang, Y., Liu, M., Pitas, I., 2021. Rethinking road surface 3 -d reconstruction and pothole detection: from perspective 
transformation to disparity map segmentation. IEEE Transactions on Cybernetics , 1-10doi:10.1109/TCYB. 2021.3060461.

Gong, F., Yue, H., Yuan, X., Gong, W., Song, T., 2019. Discriminative correlation filter for long-time tracking. The Computer Journal 63, 460468. doi:10.1093/comjnl/bxz049.

Imine, H., M'Sirdi, N., 2006. Road profile input estimation in vehicle dynamics simulation. Vehicle System Dynamics 44, 285-303. doi:10. 1080/00423110500333840.

Kalman, R.E., 1960. A New Approach to Linear Filtering and Prediction Problems. Journal of Basic Engineering 82, 35-45. doi:10.1115/1. 3662552.

Kang, S.W., Kim, J.S., Kim, G.W., 2019. Road roughness estimation based on discrete Kalman filter with unknown input. Vehicle System Dynamics 57, 1530-1544. doi:10.1080/00423114.2018.1524151.

Kok, V., Mpofu, N., Olusanya, M., 2021. Severity estimation of potholes in imagery using convolutional neural networks, in: Silhavy, R. (Ed.), Artificial Intelligence in Intelligent Systems, Springer International Publishing, Online. pp. 658-672. doi:10.1007/978-3-030-77445-5_60.

Li, Y., Papachristou, C., Weyer, D., 2018. Road pothole detection system based on stereo vision, in: NAECON 2018 - IEEE National Aerospace and Electronics Conference, IEEE, Dayton, OH, USA. pp. 292-297. doi:10.1109/NAECON. 2018.8556809.

Li, Z., Kalabić, U.V., Kolmanovsky, I.V., Atkins, E.M., Lu, J., Filev, D.P., 2016. Simultaneous road profile estimation and anomaly detection with an input observer and a jump diffusion process estimator, in: 2016 American Control Conference (ACC), pp. 1693-1698. doi:10.1109/ACC. 2016.7525160.

Nguyen, T., Lechner, B., Wong, Y.D., 2019. Response-based methods to measure road surface irregularity: A state-of-the-art review. European Transport Research Review 11, 43. doi:10.1186/s12544-019-0380-6.

Rath, J.J., Veluvolu, K.C., Defoort, M., 2014. Estimation of road profile for suspension systems using adaptive super-twisting observer, in: 2014 European Control Conference (ECC), pp. 1675-1680. doi:10.1109/ECC. 2014.6862248.

Reimpell, J., Stoll, H., Betzler, J., 2001. The automotive chassis: engineering principles. 2nd ed., Elsevier.

U1 Haq, M.U., Ashfaque, M., Mathavan, S., Kamal, K., Ahmed, A., 2019. Stereo-based 3D reconstruction of potholes by a hybrid, dense matching scheme. IEEE Sensors Journal 19, 3807-3817. doi:10.1109/JSEN. 2019. 2898375.

Vupparaboina, K.K., Tamboli, R.R., Shenu, P.M., Jana, S., 2015. Laserbased detection and depth estimation of dry and water-filled potholes: A geometric approach, in: 2015 Twenty First National Conference on Communications (NCC), pp. 1-6. doi:10.1109/NCC. 2015.7084929.

Wang, J., Song, Q., Jiang, Z., Zhou, Z., 2016. A novel InSAR based offroad positive and negative obstacle detection technique for unmanned ground vehicle, in: 2016 IEEE International Geoscience and Remote Sensing Symposium (IGARSS), pp. 1174-1177. doi:10.1109/IGARSS. 2016.7729297.

Wong, J.Y., 2008. Theory of ground vehicles. 4 ed., John Wiley \& Sons.

Yang, J.N., Pan, S., Huang, H., 2007. An adaptive extended Kalman filter for structural damage identifications II: Unknown inputs. Structural Control and Health Monitoring 14, 497-521. doi:10.1002/stc. 171.

Yu, Z., 2018. Automobile theory. 6 ed., China Machine Press.

Zhao, B., Nagayama, T., Xue, K., 2019a. Road profile estimation, and its numerical and experimental validation, by smartphone measurement of the dynamic responses of an ordinary vehicle. Journal of Sound and Vibration 457, 92-117. doi:10.1016/j.jsv. 2019.05.015.

Zhao, X., Wu, X., Sun, Y.E., Huang, H., Du, Y., Cao, Z., 2019b. CPDM: An efficient crowdsensing-based pothole detection and measurement system design, in: 2019 IEEE 31st International Conference on Tools with Artificial Intelligence (ICTAI), IEEE, Portland, OR, USA. pp. 547554. doi:10.1109/ICTAI. 2019.00082.

Zhong, W., Suo, Q., Ma, F., Hou, Y., Gupta, A., Qiao, C., Su, L., 2019. A reliability-aware vehicular crowdsensing system for pothole profiling. Proceedings of the ACM on Interactive, Mobile, Wearable and Ubiquitous Technologies 3, 160:1-160:26. doi:10.1145/3369815. 